FP8 (continued)

healthy recipes with their families. The core outcome is the ability to prepare a healthy meal or dish based on USDA MyPlate guidelines.

Evaluation: Pre- and post-surveys will be analyzed for all children who participate in Farmers Grow MyPlate and Power Chef Challenge. They will be scored, and pre- and post-intervention scores will be compared using t-tests.

Conclusions and Implications: Survey results from the 2014-2016 implementation of Farmers Grow MyPlate will be shared. Pilot program survey results from the spring 2017 implementation of Power Chef Challenge will also be shared. Process measures will inform modifications to both Farmers Grow MyPlate and Power Chef Challenge.

Funding: USDA Team Nutrition Training Grant, FNSCNTN-16-SD, FNS-CNTN-14-SD

\section{FP9 Connecticut Fitness and Nutrition Clubs In Motion-Reflection and Future of 4-H STEM in the Prevention of Childhood Obesity}

Umekia R. Taylor, RD, MS, RDN, CDN, umekia.taylor@ uconn.edu, UConn Extension, 305 Skiff Street, North Haven, CT 06473; Miriah Russo Kelly, PhD, UConn Extension, College of Agriculture, Health and Natural Resources; Linda Castro, MPH; Lindsey C. Brush, BS; Danielle Annett, PhD, UConn Center for Applied Research, Human Development and Family Studies; Barbara Chamberlin, PhD, New Mexico State University, College of Agriculture, Environmental and Consumer Sciences, Media Productions; Amy Smith Muise, New Mexico State University, Multi Media Productions, Learning Games Lab; Sandra Johnson, PhD, New Mexico State University, Instructional Innovation and Quality; Susan Bussman; New Mexico State University, Instructional Innovation and Quality

Objective: To reflect upon impacts of CT FANs 4-H STEM Afterschool (CT FANs IM) among third and fourth graders, their school, families, and teen mentors.

Description: CT FANs IM has been established in five cities of three counties over a five-year span, supported by a curriculum and leaders' training guide delivered online. An interactive app and cooking videos have been developed and introduced to parents and children; the recipes include produce that can be garden grown.

Evaluation: Evaluation has included iPad-based Qualtrics questionnaires and fitness measures for a small subset study. Qualitative interviews have been initiated with current and sustained CT FANs IM sites (youth, parents, teachers, teen mentors). Preliminary teacher input regarding the online curriculum has been favorable. Parent and child teams participated in formative evaluation of the interactive app and cooking videos, and their responses guided design. Follow up interviews with parents suggested at least half of the children later cooked a recipe at home.

Conclusions and Implications: Despite the limited intervention time, data suggests a positive influence of behaviors, attitudes, healthy eating, fitness and self-efficacy. The sustainability of CT FANs IM, 4-H STEM programming and gardening has been interwoven with committed school staff and volunteers. For the remainder of the grant, the team will promote the online CT FANs IM curriculum, cooking videos and interactive app via 4-H, EFNEP and current partners and families. These outputs will help to extend the life and influence in prevention of childhood obesity via CT FANs IM.

Funding: USDA Team Nutrition Training Grant, 201268001-19956

\section{FP10 Idaho Grown Legumes - Chef Recipes for Child Nutrition Programs}

Brenda Thompson-Wattles, LDN, RD, bthompsonwattles@ sde.idaho.gov, Chef, Idaho Department of Education, 650 West State Street, Boise, ID 83702

Objective: The Idaho Grown Legumes-Chef Recipes for Child Nutrition Programs cookbook was designed to promote legumes in the Child Nutrition Programs (CNP), provide culinary techniques on preparing legumes, offer recipes that appeal to children's tastes, and meet the meal patterns for grades K-12.

Description: The Idaho Grown Legumes-Chef Recipes for Child Nutrition Programs cookbook was developed by a culinary chef and registered dietitian. The cookbook provided legume taste tests to 1,589 K-12 students throughout Idaho. Students tasted and voted on 16 legume recipes that met the National School Lunch Program Meal Pattern. The cookbook includes a variety of training resources including an explanation of legume health benefits; tips and tricks for preparing and cooking legumes; how to credit legumes to the NSLP Lunch Meal Pattern; how to conduct taste testing; and methods of food promotion in schools.

Evaluation: This project allowed children to select their own healthy choices through an evidence-based strategy: taste testing. According to Cornell University's Center for Behavior Economics in Child Nutrition Programs, a child who is given a choice between two healthy foods will continue to choose healthier foods simply because they have the ability to decide.

Conclusions and Implications: The Idaho Grown Legumes-Chef Recipes for Child Nutrition Programs cookbook promotes the legume vegetable subgroup to Idaho's children. Students evaluated 16 legume recipes, by voting "I like it" "It's okay" or "I don't like it" for each recipe. Taking the average score for the top ten recipes resulted in $74 \%$ "Like," 18\% "Okay" and 8\% "dislike" ratings. All 16 recipes were compiled into the cookbook and the student evaluation results were listed for each recipe.

Funding: USDA Team Nutrition Training Grant, CNTN14-ID USDA FNS Team Nutrition

\section{FP11 WISEly Improving Preschoolers Fruit and Vegetable Consumption at Home}

Leanne Whiteside-Mansell, EdD, UAMS; Taren Swindle, PhD; Danya Johnson, BA, JohnsonDanyaL@uams.edu, 521 Jack Stephens Drive, Little Rock, Arkansas 72205 